\title{
NGC 7419: An open cluster rich in Be stars
}

\author{
A. Pigulski and G. Kopacki \\ Wrocław University Observatory, Kopernika 11, 51-622 Wrocław, Poland \\ e-mail: pigulski, kopacki@astro.uni.wroc.pl
}

Received July 27; accepted August 13, 2000

\begin{abstract}
The results of our CCD photometric $\mathrm{H} \alpha$ observations of NGC 7419 are presented. The observations resulted in a discovery of 17 new Be stars and two other emission-line objects. In consequence, the number of known Be stars in this young cluster increased more than twofold and equals now to 31 . This is at present the largest number of Be stars known in any galactic open cluster. Moreover, we estimate that these 31 Be stars constitute $36 \pm 7 \%$ of all cluster B-type stars brighter than $R_{\mathrm{C}}=$ 16.1 mag. This locates NGC 7419 among these open clusters which are richest in Be stars such as galactic NGC 663, NGC 330 in SMC, and NGC 1818A in LMC.
\end{abstract}

Key words: stars: emission-line, Be - open clusters and associations: individual: NGC 7419

\section{Introduction}

In a debate on the fraction of Be stars in young open clusters it is often forgotten that, in fact, a relatively small number of clusters has been deeply searched for the presence of Be stars. The objective-prism spectroscopic surveys which led to the discovery of the most of known Be stars are magnitude-limited and ineffective in crowded regions. In consequence, our knowledge of Be star fraction in clusters is usually confined to the brightest, that is, the early B-type stars. It is therefore possible that clusters with large number of Be stars could still be discovered. The use of CCD detectors and the $\mathrm{H} \alpha$ filters opens a convenient way to fill in this gap, allowing both the crowdedfield photometry and the detection of $\mathrm{H} \alpha$ emission even in late B-type stars. With a moderate-size telescope even Magallanic Clouds clusters are within reach (Grebel 1997; Keller et al. 1999).

Among the galactic open clusters there are three that are usually mentioned as those which contain large number of Be stars. These are $\chi$ Persei, NGC 3766, and

Send offprint requests to: A. Pigulski
NGC 663 (Mermilliod 1982; Slettebak 1985). Of these, NGC 663 is the richest in Be stars since about $35 \%$ of its B stars show $\mathrm{H} \alpha$ emission (Maeder et al. 1999). In the percentage of Be stars, NGC 663 is surpassed only by such Magellanic Clouds clusters as NGC 330 and NGC 1818A (Keller et al. 1999).

In the present paper we report results of our CCD $\mathrm{H} \alpha$ photometry of the highly reddened galactic cluster NGC 7419. We find in the cluster a large number of previously unknown Be stars.

\section{Observations and reductions}

All photometric observations of NGC 7419 were carried out at the Białków Observatory of the Wrocław University using the $60-\mathrm{cm}$ reflecting telescope equipped with a $576 \times 384$ pixels CCD camera covering a $6^{\prime} \times 4^{\prime}$ field of view. In order to detect emission in the $\mathrm{H} \alpha$ line, we used two interference filters centred on this line with the full width at half maximum of 20 and $3 \mathrm{~nm}$ for wide and narrow $\mathrm{H} \alpha$ filter, respectively.

The H $\alpha$ observations of NGC 7419 were carried out on a single night of November 15, 1999. We took 11 frames in the wide $\mathrm{H} \alpha$ filter and 8 frames in the narrow one with the total integration time of 6600 and $18500 \mathrm{~s}$, respectively. The $\mathrm{H} \alpha$ observations were supplemented by observations of the same field through Cousins $R_{\mathrm{C}}$ and $I_{\mathrm{C}}$ filters made on November $15,1999\left(R_{\mathrm{C}}\right.$, a single 600 -s frame $)$ and September 17, 1999 and January 6, $2000\left(I_{\mathrm{C}}\right.$, twenty 120-s frames). These observations were transformed to the standard system using the photometry of Bhatt et al. (1993), tied to the Landolt (1983) standards. We do not give here the details of transformations and present the photometry in the graphical form only. They are, however, available from the authors upon request. The resulting colourmagnitude diagram (CMD) shown in Fig. 4 is used for distinguishing some of the non-members. All stars which were detected both in the $R_{\mathrm{C}}$ and $I_{\mathrm{C}}$-filter frames are shown in Fig. 1. 


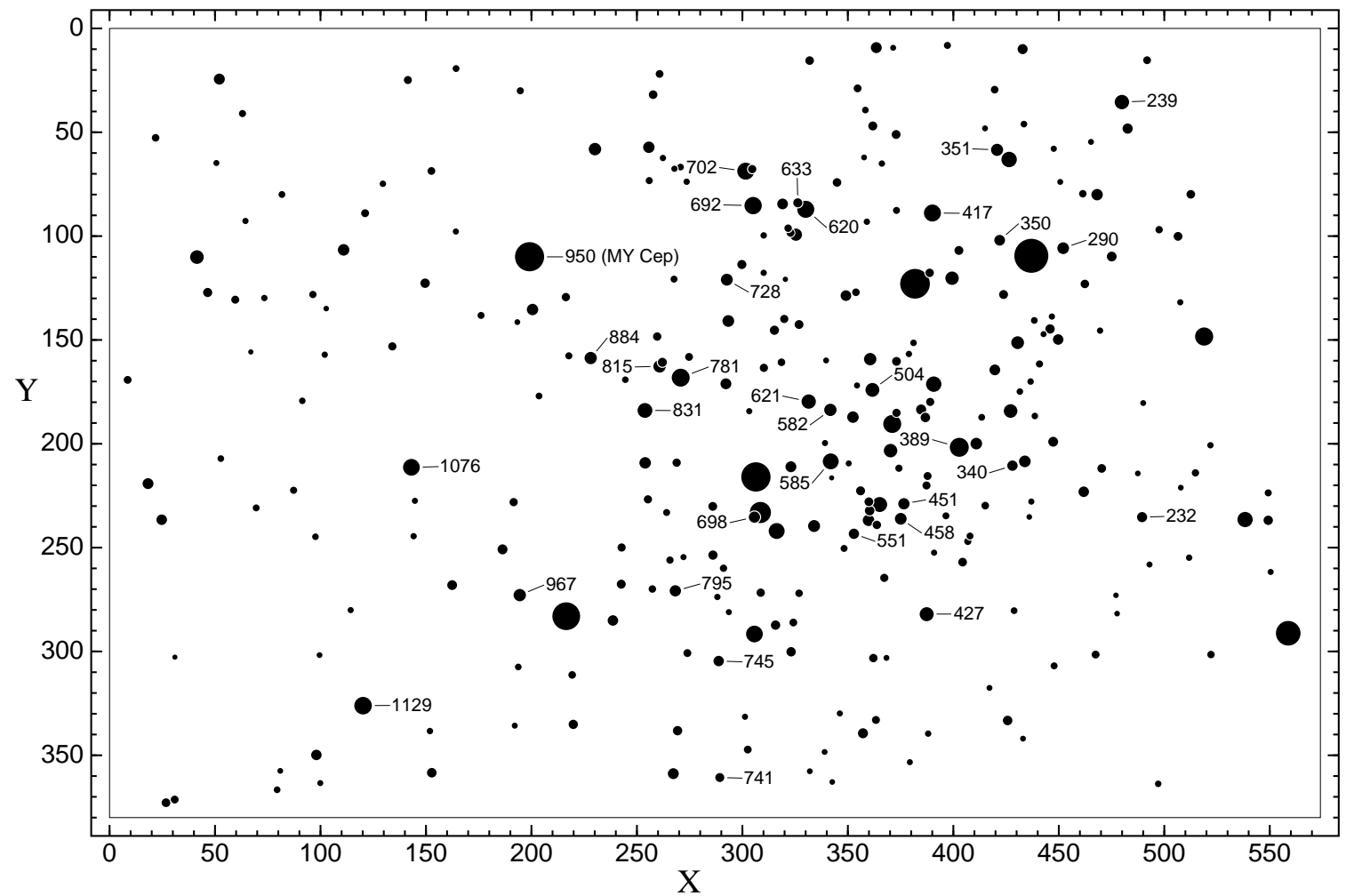

Fig. 1. Schematic view of our $6^{\prime} \times 4^{\prime}$ field in NGC 7419. The diameters of the circles were chosen to resemble the cluster appearance as seen through the $R_{\mathrm{C}}$ filter. All emission-line objects listed in Table 1 are labeled with numbers from B94. North is up, east to the left

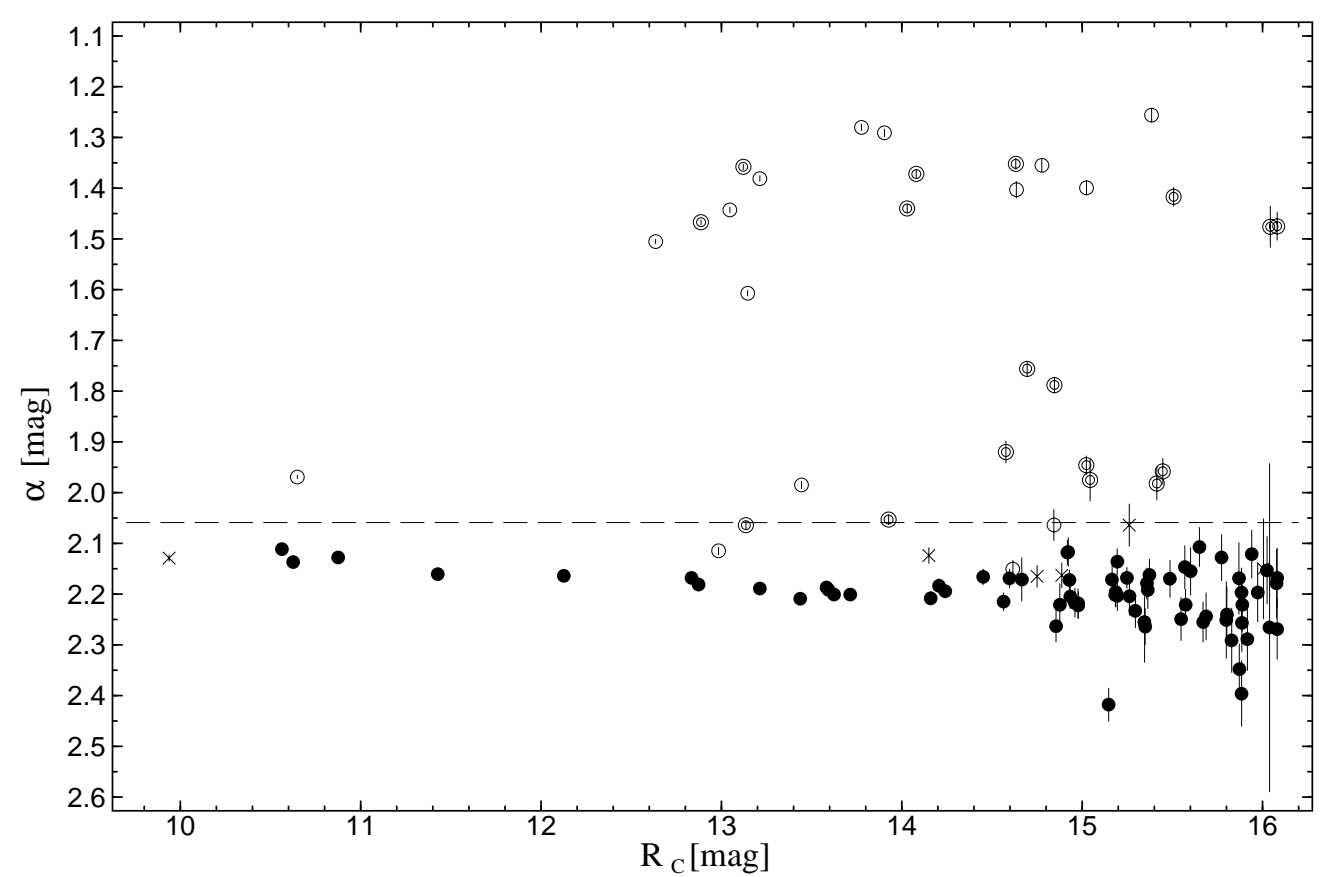

Fig. 2. The $\alpha$ index (in mag) for 107 stars brighter than $R_{\mathrm{C}}=16.1 \mathrm{mag}$, plotted as a function of $R_{\mathrm{C}}$. Non-members are shown with crosses, cluster members showing $\mathrm{H} \alpha$ emission, with open circles (the circles are double for new Be stars), remaining stars, with filled circles. The dashed line corresponds to the expected value of $\alpha$ for zero equivalent width of the $\mathrm{H} \alpha$ line 
Table 1. Stars in NGC 7419 showing H $\alpha$ emission. The columns are: (1): Beauchamp et al. (1994) number, (2): Bhatt et al. (1993) number, E precedes the number of a star in the eastern region of the cluster, W, a star in the western region, (3): Kohoutek \& Wehmeyer (1997) number, (4) and (5): $X$ and $Y$ coordinate in Fig. 1, (6): right ascension (epoch 2000.0, in hours, minutes, and seconds), (7): declination (epoch 2000.0, in degrees, arcmin and arcsec), (8): $R_{\mathrm{C}}$ magnitude (in mag), $(9):(R-I)_{\mathrm{C}}$ colour index (in mag), (10): $\alpha$ index (in mag), (11): remarks

\begin{tabular}{|c|c|c|c|c|c|c|c|c|c|c|}
\hline BMD & BPMM & $\mathrm{HBH}$ & $X$ & $Y$ & Right Asc. & Decl. & $R_{\mathrm{C}}$ & $(R-I)_{\mathrm{C}}$ & $\alpha$ & Remarks \\
\hline (1) & $(2)$ & (3) & $(4)$ & (5) & (6) & (7) & (8) & (9) & (10) & (11) \\
\hline 232 & W12 & - & 489.5 & 235.4 & 225407.10 & +604817.3 & 15.51 & 1.33 & $1.417 \pm 0.019$ & new \\
\hline 239 & W14 & 6206-14 & 479.9 & 35.5 & 225407.55 & +605022.7 & 13.90 & 1.31 & $1.291 \pm 0.008$ & \\
\hline 290 & W23 & - & 452.0 & 105.8 & 225410.05 & +604938.9 & 14.84 & 1.18 & $2.064 \pm 0.031$ & D 22-117: \\
\hline 340 & W33 & - & 428.1 & 210.6 & 225412.29 & +604833.6 & 15.41 & 1.20 & $1.982 \pm 0.033$ & new \\
\hline 351 & W37 & - & 420.8 & 58.5 & 225412.63 & +605009.0 & 14.70 & 1.15 & $1.756 \pm 0.015$ & new \\
\hline 389 & W45 & 6206-13 & 402.8 & 201.7 & 225414.42 & +604839.5 & 12.64 & 1.14 & $1.505 \pm 0.005$ & B1-2 I:e (B94) \\
\hline 417 & - & - & 390.1 & 88.9 & $2254 \quad 15.30$ & +604950.4 & 13.14 & 1.06 & $2.064 \pm 0.008$ & new \\
\hline 427 & W54 & - & 387.4 & 282.0 & 225415.89 & +604749.4 & 14.03 & 1.23 & $1.440 \pm 0.008$ & new \\
\hline 451 & W58 & - & 376.6 & 228.9 & $2254 \quad 16.71$ & +604822.8 & 15.02 & 1.08 & $1.946 \pm 0.019$ & new \\
\hline 458 & W60 & - & 375.0 & 236.1 & 225416.85 & +604818.3 & 14.85 & 1.12 & $1.788 \pm 0.016$ & new \\
\hline 504 & E10 & - & 361.6 & 174.0 & 225417.88 & +604857.4 & 14.08 & 1.11 & $1.372 \pm 0.008$ & new \\
\hline 551 & E20 & - & 352.9 & 243.3 & 225418.75 & +604814.1 & 15.45 & 1.08 & $1.958 \pm 0.026$ & new \\
\hline 582 & $\mathrm{E} 25$ & - & 341.7 & 183.7 & 225419.59 & +604851.6 & 14.63 & 1.02 & $1.352 \pm 0.011$ & new \\
\hline 585 & $\mathrm{E} 26$ & $6206-12$ & 341.9 & 208.4 & 225419.62 & +604836.1 & 13.44 & 1.02 & $1.985 \pm 0.008$ & \\
\hline 620 & E30 & - & 330.1 & 87.1 & 225420.41 & +604952.3 & 13.12 & 1.14 & $1.358 \pm 0.006$ & new \\
\hline 621 & E31 & - & 331.4 & 179.7 & 225420.46 & +604854.3 & 13.93 & 1.12 & $2.053 \pm 0.010$ & new \\
\hline 633 & - & - & 326.3 & 84.0 & 225420.73 & +604954.3 & 16.04 & 1.10 & $1.476 \pm 0.041$ & new \\
\hline 692 & E50 & 6206-11 & 305.1 & 85.3 & 225422.54 & +604953.7 & 13.05 & 1.15 & $1.443 \pm 0.006$ & \\
\hline 698 & - & - & 305.7 & 235.3 & 225422.76 & +604819.7 & 15.04 & 1.04 & $1.975 \pm 0.042$ & new \\
\hline 702 & E53 & $6206-18$ & 301.6 & 68.8 & 225422.81 & +605004.1 & 13.15 & 1.13 & $1.607 \pm 0.006$ & \\
\hline 728 & $\mathrm{E} 57$ & $6206-15$ & 292.6 & 121.0 & 225423.67 & +604931.5 & 14.78 & 1.01 & $1.355 \pm 0.013$ & \\
\hline 741 & - & - & 289.3 & 360.7 & 225424.37 & +604701.3 & 16.08 & 1.21 & $1.475 \pm 0.028$ & new \\
\hline 745 & E61 & $6206-10$ & 288.7 & 304.6 & 225424.32 & +604736.5 & 15.39 & 1.18 & $1.256 \pm 0.014$ & \\
\hline 781 & E68 & - & 270.8 & 168.2 & 225425.61 & +604902.3 & 12.89 & 1.09 & $1.467 \pm 0.005$ & new, B1-2 I: (B94) \\
\hline 795 & E71 & 6206-09 & 268.3 & 270.9 & 225426.01 & +604757.9 & 15.02 & 1.17 & $1.399 \pm 0.015$ & \\
\hline 815 & E75 & - & 260.7 & 162.7 & 225426.46 & +604905.8 & 14.58 & 1.02 & $1.920 \pm 0.022$ & new \\
\hline 831 & $\mathrm{E} 82$ & $6206-08$ & 253.8 & 183.9 & 225427.08 & +604852.6 & 13.78 & 1.03 & $1.280 \pm 0.007$ & \\
\hline 884 & E90 & $6206-07$ & 228.1 & 158.8 & 225429.24 & +604908.7 & 14.64 & 1.07 & $1.403 \pm 0.017$ & \\
\hline 967 & E100 & $6206-16$ & 194.5 & 272.9 & 225432.30 & +604757.6 & 14.62 & 1.07 & $2.151 \pm 0.018$ & \\
\hline 1076 & - & $6206-17$ & 143.1 & 211.4 & 225436.56 & +604836.9 & 13.21 & 1.15 & $1.381 \pm 0.006$ & D $22-124$ \\
\hline 1129 & - & - & 120.2 & 326.1 & 225438.72 & +604725.3 & 12.98 & 1.06 & $2.115 \pm 0.008$ & D $22-125$ \\
\hline 950 & E96 & - & 199.1 & 110.0 & 225431.62 & +604939.7 & 10.65 & 2.95 & $1.969 \pm 0.004$ & MY Cep \\
\hline 350 & W36 & - & 422.0 & 101.9 & 225412.60 & +604941.8 & 15.26 & 1.73 & $2.064 \pm 0.042$ & non-member \\
\hline
\end{tabular}

\section{The cluster}

NGC 7419 (C 2252+605) is a small, young open cluster in Cepheus, known mainly for its five M-type supergiants (Blanco et al. 1955; Fawley \& Cohen 1974; Beauchamp et al. 1994, henceforth B94). The coolest of them is MY Cephei $=$ IRAS 22525+6033, an Lb or SRc-type variable of spectral type M7.5 I, known also to be an $\mathrm{H}_{2} \mathrm{O}$ maser (Dickinson et al. 1978) and an $\mathrm{OH} / \mathrm{IR}$ source (Alcolea et al. 1990). The age of NGC 7419 was estimated by Bhatt et al. (1993) as smaller than $40 \mathrm{Myr}$, while B94 derived that it is equal to $14 \pm 2 \mathrm{Myr}$. The average reddening, in terms of $E(B-V)$, amounts to about $2.0 \mathrm{mag}$, but a differential effect of the order of $0.4 \mathrm{mag}$ is also observed (B94). The average total absorption in the $V$ band, as estimated by the same authors, amounts to about $6.7 \mathrm{mag}$.

\section{Be stars in NGC 7419}

The first two Be stars in NGC 7419 were discovered by González \& González (1956) and designated in their list as stars Nos. 112 and 113. Dolidze (1959) increased this number to seven. These seven stars appear also in his later compilation (Dolidze 1975). Unfortunately, finding charts in the above-mentioned papers are not detailed enough, so that only two out of the seven stars, D 22-124 and D 22-125 (Dolidze 1975), can be unambiguously identified. 


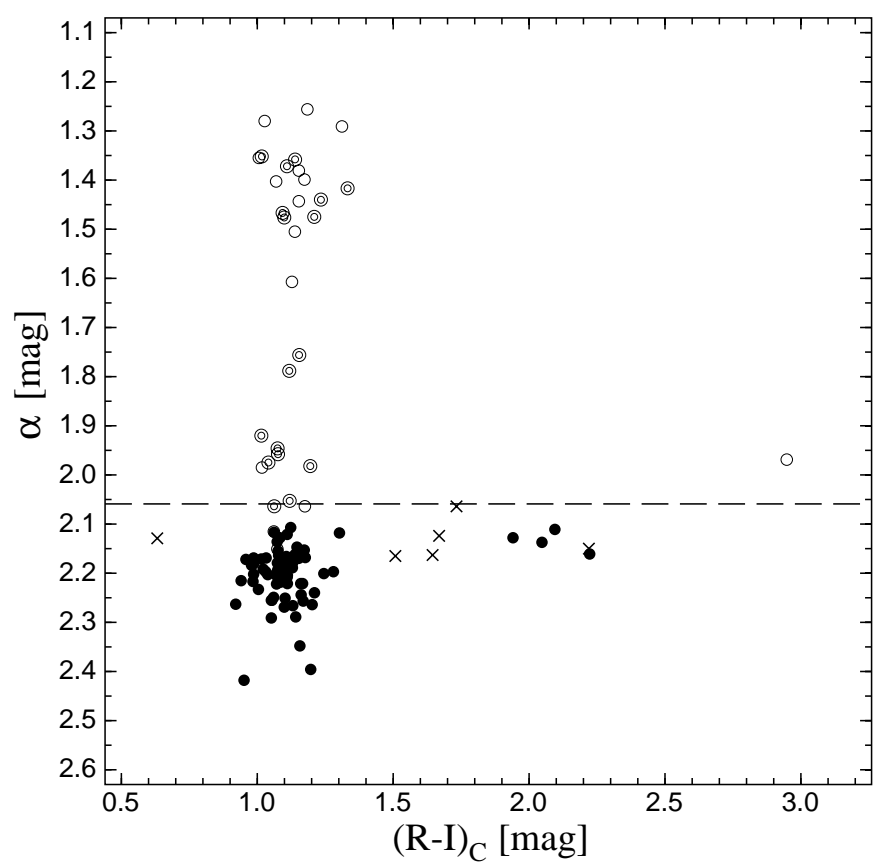

Fig. 3. The same as in Fig. 2 but plotted as a function of $(R-I)_{\mathrm{C}}$ colour. Symbols and the dashed line have the same meaning as in Fig. 2. For clarity, the error bars were omitted here

B94 found emission in one of the five brightest blue members, BMD 389 (we shall use the numbering system of B94 preceding their number by "BMD"). Recently, Kohoutek \& Wehmeyer (1997) detected 12 Be stars in the cluster, but list also two others. Altogether, 14 Be stars were known in NGC 7419 prior to our study.

Our discovery of stars showing emission in $\mathrm{H} \alpha$ line was made by means of the $\alpha$ index defined by two $\mathrm{H} \alpha$ filters described in Sect. 2, in analogy to the well-known photometric $\beta$ index, namely:

$\alpha=m_{\text {narrow }}-m_{\text {wide }}+C$,

where $m_{\text {narrow }}$ and $m_{\text {wide }}$ denote the magnitude of a star as seen through the narrow and wide $\mathrm{H} \alpha$ filter, respectively. The constant $C$ takes into account the differences in the exposure times and differences in zero points between frames.

The $\alpha$ index is shown in Fig. 2 for 107 brightest stars in the field down to magnitude $R_{\mathrm{C}}=16.1$. Only one of the previously known 14 Be stars, BMD $967=$ HBH 620616 , shows no or only weak emission. A weakening of the emission was observed in many Be stars, and this could be such a case. Remaining 13 stars stand clearly above the sequence of non-emission stars (see Fig. 2). In order to be able better recognize emission stars, especially those of late type, the $\alpha$ index was also plotted as a function of colour index $(R-I)_{\mathrm{C}}$ (Fig. 3).

In addition to the 14 emission-line objects in NGC 7419 listed by Kohoutek \& Wehmeyer (1997), 19 stars showing emission were found. Out of them, two are of late type.

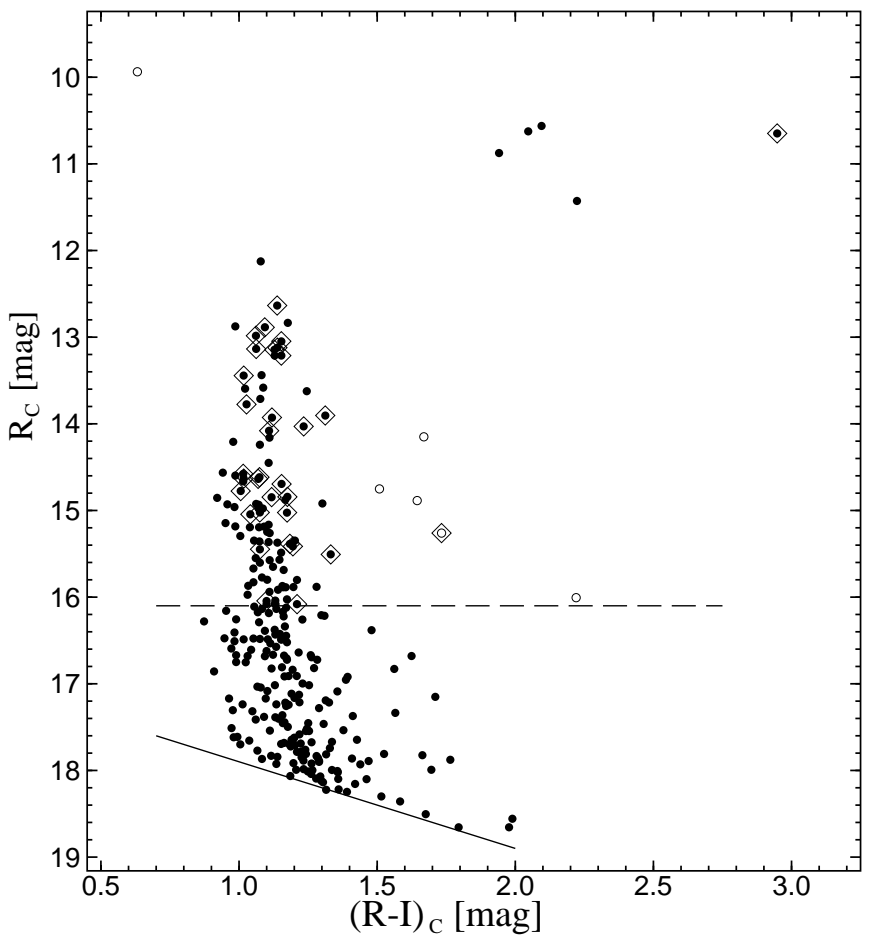

Fig. 4. CMD for NGC 7419. Open circles denote stars brighter than $R_{\mathrm{C}}=16.1 \mathrm{mag}$ which are too red or too blue to be members. Diamonds are used to indicate the emission-line objects. The solid line shows the limit of our $(R I)_{\mathrm{C}}$ photometry while the dashed one, that of the $\mathrm{H} \alpha$ photometry

The first one is MY Cep = BMD 950 mentioned in Sect. 3 . The other one, BMD 350, probably also of late type, is not a member as can be judged from its position in the cluster CMD (Fig. 4). Thus, there are now 31 stars in the observed field which are very likely cluster Be stars.

The data for all 33 stars in the observed field showing $\mathrm{H} \alpha$ emission are given in Table 1 . The typical error of $R_{\mathrm{C}}$ magnitude given in Table 1 equals to 0.004 and 0.011 mag for a star with $R_{\mathrm{C}}=13$ and 16 mag, respectively. The corresponding errors of colour indexes for such stars are 0.007 and $0.035 \mathrm{mag}$. The coordinates of stars were derived using the positions of 10 stars in the field from the NASA Guide Star Catalog, v. 1.1. They are accurate to within $0.5^{\prime \prime}$.

As can be seen in the cluster CMD (Fig. 4), the Be stars are $0.1-0.2 \mathrm{mag}$ redder in $(R-I)_{\mathrm{C}}$ than nonemission stars of the same magnitude. This is a well-known effect observed in many open clusters with Be stars.

\section{Fraction of Be stars}

Maeder et al. (1999) compare fractions of Be stars in clusters of similar age in three different intervals of $M_{V}$. The widest interval, $-5<M_{V}<-1.4$, includes all stars of type B3 and earlier. Taking $V-M_{V}=18.5 \mathrm{mag}$ for NGC 7419 (B94) and adopting average $V-R_{\mathrm{C}} \approx 1.0 \mathrm{mag}$ 
for the cluster upper main-sequence estimated from photometry of Bhatt et al. (1993), we find that this interval of $M_{V}$ corresponds roughly to $12.5<R_{\mathrm{C}}<16.1$. There are 100 stars in our field falling within this interval, of which 31 are Be stars. It is rather unlikely that any of the Be stars is a non-member, but the total number of stars in the interval is certainly affected by contamination by field stars.

In order to estimate the number of field stars in the above-mentioned interval of $R_{\mathrm{C}}$, four fields, situated about $7^{\prime}$ north, east, south and west of the cluster were observed on June 8,2000 through the $R_{\mathrm{C}}$ and $I_{\mathrm{C}}$ filters. In total, 52 stars having $12.5<R_{\mathrm{C}}<16.1$ were found in these fields. Hence, in the vicinity of NGC 7419 there are $52 / 4=13$ stars in this interval and in a $6^{\prime} \times 4^{\prime}$ field of view. All of them are probably foreground and/or background stars, because at a distance of $7^{\prime}$ from the centre of NGC7419 the percentage of cluster stars is negligible. The final estimate of the fraction of Be stars in NGC 7419 is therefore $31 /(100-13) \approx 36 \pm 7 \%$, where the error was calculated assuming Poisson statistics. If we split the above interval into two parts, i.e. $12.5<R_{\mathrm{C}}<$ 14.3 and $14.3<R_{\mathrm{C}}<16.1$, the fractions of Be stars are equal to $61 \pm 21 \%$ and $27 \pm 7 \%$, respectively.

We note that owing to the intermittency of the Be phenomenon and the fact that weak emission cannot be detected photometrically, the above estimated fraction of Be stars in NGC7419 represents a lower limit. Since the age of NGC7419 is similar to the ages of clusters investigated by Maeder et al. (1999), the fraction of $\mathrm{Be}$ stars we derived can be directly compared with their numbers. This comparison locates NGC 7419 among the clusters with largest known fractions of Be stars, such as the galactic NGC 663 (34 \pm $11 \%)$, NGC 330 in SMC (39 $\pm 7 \%)$, and NGC 1818A in LMC $(36 \pm 7 \%)$.

Acknowledgements. We thank Prof. M. Jerzykiewicz for critical reading the manuscript and Z. Kołaczkowski for his help in observations and valuable comments. This work was made with the help of Aladin Sky Atlas operating at CDS, Strasbourg.

\section{References}

Alcolea J., Bujarrabal V., Gómez-González J., 1990, A\&A 231, 431

Beauchamp A., Moffat A.F.J., Drissen L., 1994, ApJS 93, 187 (B94)

Bhatt B.C., Pandey A.K., Mohan V., Mahra H.S., Paliwal D.C., 1993, Bull. Astron. Soc. India 21, 33

Blanco V., Nassau J.J., Stock J., Wehlau W., 1955, ApJ 121, 637

Dickinson D.F., Blair G.N., Davis J.H., Cohen N.L., 1978, AJ 83,32

Dolidze M.V., 1959, Bull. Abastumani Astron. Obs. 24, 7

Dolidze M.V., 1975, Bull. Abastumani Astron. Obs. 47, 3

Fawley W.M., Cohen M., 1974, ApJ 193, 367

González G., González G., 1956, Boletin de los Observatorios Tonantzintla y Tacubaya 15, 16

Grebel E.K., 1997, A\&A 317, 448

Keller S.C., Wood P.R., Bessell M.S., 1999, A\&AS 134, 489

Kohoutek L., Wehmeyer R., 1997, Abhandlungen aus der Hamburger Sternwarte, Band XI

Landolt A.U., 1983, AJ 88, 439

Maeder A., Grebel E.K., Mermilliod J.-C., 1999, A\&A 346, 459

Mermilliod J.-C., 1982, A\&A 109, 48

Slettebak A., 1985, ApJS 59, 769 